AUDIT REPORT

\title{
UK National Audit of Early Syphilis Management. Clinics Audit: Screening for and Management of Early Syphilis
}

\author{
Hugo McClean FRCPI, David Daniels FRCP, Chris Carne MD FRCP, Paul Bunting MSc, Rob \\ Miller FRCP
}

\section{on behalf of the National Audit Group of the British Association for Sexual Health and HIV}

Summary: Data were provided by 131 clinics, and $56 \%$ of cases were managed in clinics in the London Regions in 2003. Three clinics (2\%) do not routinely screen new patients for syphilis, and 28 clinics $(21 \%)$ do not routinely screen "rebook" patients who have had a new partner. More than $80 \%$ of clinics routinely conduct cardiovascular and neurological examinations, although chest radiography is only performed by $50 \%$ of clinics and lumbar puncture by $13 \%$. Only 19 (14\%) clinics indicated not routinely using the recommended procaine penicillin G (PPG) regimen or one or two dose benzathine penicillin G (BPG) regimens for early syphilis, with $57 \%$ providing two doses of BPG 2.4 grams, 40\% providing PPG $750 \mathrm{mg}$ for 10 days, and 15\% providing one dose of BPG 2.4 grams. Only 7 clinics (5\%) indicated that they provided treatment for early syphilis with PPG that is inferior to that recommended in the National Guidelines. In cases with HIV infection, the recommended treatments for early syphilis with PPG and/or one or two dose BPG were used in $51(39 \%)$ clinics and, of these, 38 (75\%) clinics specified PPG regimens in excess of that recommended by the National Guidelines. Provision for management of severe penicillin reaction is good, although few patients are desensitised. All clinics report that contact tracing for early syphilis is provided, and is mainly the responsibility of health advisers. Compared to auditing outcomes, audit of management policies overestimated performance in contact tracing and provision of dark ground microscopy.

Keywords (MeSH): Syphilis, Medical Audit, Nursing Audit, Syphilis Serodiagnosis, Drug Therapy, Contact Tracing, Drug Hypersensitivity 


\section{Introduction}

This paper accompanies the two papers that reported on case note auditing in the UK National Audit of Early Syphilis Management, carried out in 2004 by the National Audit Group in response to increasing numbers of cases of infectious syphilis in the UK. ${ }^{1,2}$

\section{Methods}

The Audit methodology and organisation are described in the report on diagnosis and treatment. ${ }^{1}$ This part of the audit describes national averages and regional ranges for measurements of various aspects of clinical management in 2003. The results in this paper are derived from questionnaires sent to the lead clinicians in all genitourinary medicine clinics in the UK. The Questionnaire on Screening for and Management of Early Syphilis is available on the BASHH website (http://www.bashh.org/committees/nag/index.htm).

\section{Results}

Type of clinic and case load

One hundred and thirty one clinics contributed data, comprising 91 clinics from outside of the London Regions and 40 within the London Regions. The following types of clinic were recorded: district general hospitals (DGH) ( 75 clinics, $58 \%$ ), teaching hospitals ( 35 clinics, $27 \%$ ), primary care trust (PCT)/community clinic (15 clinics, $11 \%$ ), community clinic within an acute trust Medicine \& Elderly organisation (1 clinic), DGH/teaching hospital (1 clinic), teaching hospital clinic run in a health centre (1 clinic), PCT managed clinic in a hospital (1 clinic), service made up of four PCT clinics and one DGH clinic (1 clinic), and not answered (1 clinic). Numbers of both early syphilis diagnoses and annual attendances (new plus rebooked) in 2003 were returned for 109 clinics. The median percentage of annual attendances of early syphilis diagnoses for these clinics was: London clinics: $0.09 \%$ (clinic range $0.01-0.95 \%$ ), clinics outside London: $0.09 \%$ (clinic range $0.01-1.38 \%$ ) and overall: $0.09 \%$ (clinic range $0.01-1.38$. A total of 1527 cases of early syphilis were reported by participating clinics. Management took place in the following types of clinic: teaching hospitals $992(65 \%)$ cases, DGH $400(26 \%)$ cases, PCT $108(7 \%)$ cases, other types of service $18(1 \%)$ cases, and not answered $9(0.6 \%)$ cases. Overall, $862(56 \%)$ cases were managed in clinics in the London Regions. There were 65 cases of pregnant women with early syphilis and no cases of congenital syphilis. 


\section{Use of tests for screening and investigation}

Clinics reported screening the following specified patient groups for syphilis in 2003: all new patients, 128 (98\%, regional range 80-100\%); all "rebook" patients, 82 (63\%, range 0-90\%); "rebook" patients with a new sexual partner since a previous test, 103 (79\%, range 0-100\%); pregnant women, $96(73 \%$, range $0-100 \%)$. Table 1 shows the tests used to routinely screen for and investigate early syphilis in 2003. In addition, 16 clinics specified routinely screening for syphilis in patients with HIV infection.

\section{Examinations routinely performed}

The following examinations (specified in the audit questionnaire) were reported by clinics as routinely performed in investigating suspected syphilis infection: cardiovascular system examination 108 (82\%, range 50-100\%); neurological examination 107 (82\%, range 50-100\%); chest radiograph 66 (50\%, range $0-85 \%)$; ophthalmic assessment 44 (34\%, range $0-67 \%)$; electrocardiogram 34 (26\%, range 0-50\%); and lumbar puncture 17 (13\%, range $0-50 \%)$. Twenty six comments were returned. Replies from 16 clinics stated that these examinations were necessary only in latent or late syphilis, or if clinically indicated. Additionally the following comments about examinations done were recorded: "depends on the stage of infection" (2 clinics), "only in secondary, early latent and late syphilis" (1 clinic), "chest radiograph only done for patients older than 35 years" ( 1 clinic), "only if clinically indicated" ( 1 clinic). The remaining comments reported on additional examinations done: abdominal and eye examination; echocardiogram and ear, nose and throat examination and lumbar puncture if appropriate; genital and skin examination; genital and general examination; oral, rectal and skin examination (1 clinic each).

\section{Treatments routinely used to treat early syphilis}

Table 2 shows the routine use, as reported by clinics, of parenteral penicillin treatments recommended in the UK National Guidelines. ${ }^{3}$ The recommended treatments for early syphilis with procaine penicillin $\mathrm{G}$ (PPG) and/or one or two dose benzathine penicillin $G$ (BPG) were used in 112 (85\%) clinics (with 25 clinics recording use of both the recommended regimen of PPG and two dose BPG, 2 clinics the recommended regimen of PPG and one dose BPG, and 2 all three recommended regimens). Nineteen (14\%) clinics indicated not using any of the recommended PPG or BPG regimens. Of these, 4 clinics, in 4 Regions, specified using 600,000 international units (iu) of PPG (2 clinics for 10 days and 2 clinics for 14 days), 5 clinics treated early syphilis 
with $750 \mathrm{mg}$ of PPG for 14 days (4 of which also used doxycycline regimens), one clinic specified using 1.2 mega units of PPG for 10 days, one clinic specified using doxycycline $100 \mathrm{mg}$ bd for 14 days only, and one clinic "treatment according to national guidelines", and the remaining 7 clinics provided no further information. The group of clinics that used recommended PPG and/or BPG treatments also specified using non-recommended treatments: 3 clinics reported using 600,000 international units (iu) of PPG, one clinic for 14 days and the other 2 clinics for 10 days, and 2 clinics also specified treating early syphilis with $750 \mathrm{mg}$ of PPG for 14 days.

Nationally, 26 (20\%) clinics routinely used doxycycline to treat early syphilis, of which 14 (54\%) used the recommended regimen of $100 \mathrm{mg}$ bd for 14 days. The other 12 clinics used the following regimens: $100 \mathrm{mg} / 200 \mathrm{mg}$ for 14 days ( 1 clinic), $200 \mathrm{mg}$ bd for 14 days (2 clinics), $200 \mathrm{mg}$ bd for 20 days ( 1 clinic), $200 \mathrm{mg}$ bd for 28 days ( 1 clinic), $200 \mathrm{mg}$ od for 14 days ( 1 clinic), $200 \mathrm{mg}$ dosing not specified for $14,28,30$ days (2, 1, 1 clinic respectively), $300 \mathrm{mg}$ od for 21 days (2 clinics). Except for one clinic, these clinics also used the recommended PPG or BPG regimens.

Three clinics specified using PPG for epidemiological treatment with less than $750 \mathrm{mg}$ of PPG for 10 days ( 1 clinic): 600,000 iu for 10 days ( 2 clinics) and 600,000 iu for 14 days ( 1 clinic). Four clinics used $750 \mathrm{mg}$ of PPG for 14 days for epidemiological treatment. Thirteen clinics specified use of doxycycline regimens for epidemiological treatment (including four clinics that also used the recommended PPG regimen): $100 \mathrm{mg}$ bd for 14 days (6 clinics), $200 \mathrm{mg}$ bd for 14 days (1 clinic), $200 \mathrm{mg}$ bd for 28 days (2 clinics), $200 \mathrm{mg}$ dosing not specified for 28 days (1 clinic), $300 \mathrm{mg}$ od for 21 days ( 2 clinics), and not specified (1 clinic). Six clinics specified using azithromycin (including 2 clinics that also used the recommended PPG regimen), one clinic used either doxycycline or azithromycin, and one clinic specified use of national guidelines for epidemiological treatment. Two clinics specified that epidemiological treatment was not routinely offered.

In cases with HIV infection, treatments recommended for early syphilis ( $750 \mathrm{mg}$ of PPG for 10 days and/or one or two dose BPG) were used in 51 (39\%) clinics (with 6 clinics recording use of both the recommended regimen of PPG and two dose BPG. A further $51(39 \%)$ clinics indicated using a PPG or BPG regimen in HIV infection other than that recommended for early syphilis. Of these, $38(75 \%)$ clinics specified PPG regimens in excess of $750 \mathrm{mg}$ od and/or for longer than 10 days (and not represented in Table 2): 50,000 u/kg for 14 days (2 clinics), $750 \mathrm{mg}$ od for 12 days (1 clinic, this clinic also used 3 dose BPG), $750 \mathrm{mg}$ od for 14 days ( 3 clinics), $750 \mathrm{mg}$ od for 17 
days ( 7 clinics), $750 \mathrm{mg}$ od for 20 days ( 1 clinic), 1.2 mega units od for 17 days (one clinic), 1.2 mega units od for 21 days (2 clinics), 2 mega units od for 17 days (12 clinics), 2 mega units for 21 days (one clinic), 2.4 mega units od for 17 days (4 clinics), Jenacillin $A$ (dose not specified) for 17 days (one clinic), Jenacillin (preparation not specified) $8 \mathrm{mls}$ for 17 days (one clinic), Tubex (preparation not specified) $3 \mathrm{ml}$ for 17 days (one clinic) and Jenacillin $A 6.5 \mathrm{ml}$ for 21 days (one clinic). Twelve (32\%) of these clinics specified use of probenecid with PPG treatments (there was no other use of probenecid reported apart from this use for patients with HIV infection).

Thirteen (25\%) clinics used three dose BPG as the only penicillin treatment.

Of the remaining 29 (22\%) clinics, one clinic each recorded treatment as for late latent syphilis, PPG treatment with 600,000 iu od for 10 days, non-specified use of PPG, doxycycline $400 \mathrm{mg}$ (dosing not specified) for 14 days, treatment according to national guidelines and a non-specified treatment for 14 days, and 23 clinics did not report a treatment for early syphilis cases with HIV infection.

Six clinics (two of which also used a recommended PPG or BPG treatment for early syphilis) specified use of doxycycline for treatment of patients with HIV infection: $100 \mathrm{mg}$ (dosing not specified) for 21 days (2 clinics), $200 \mathrm{mg}$ (dosing not specified) for 14 days ( 1 clinic), $200 \mathrm{mg} \mathrm{bd}$ for 28 days ( 2 clinics), $200 \mathrm{mg}$ bd for 20 days ( 1 clinic), and $400 \mathrm{mg}$ (dosing not specified) for 28 days (1 clinic). Additionally, one clinic each specified use of intravenous ceftriaxone, PPG 600,000 iu od for 10 days, and $750 \mathrm{mg}$ od for 21 days (all of which also specified using a recommended early syphilis) to treat early syphilis in cases with HIV infection.

\section{Contact tracing}

All clinics reported providing contact tracing. Contact tracing was recorded as the responsibility of health advisers for 121 (92\%, range $70-100 \%)$ clinics, doctors $38(29 \%$, range $0-100 \%)$ and nurses $16(12 \%, 0-50 \%)$. One clinic recorded that a health practitioner had responsibility for contact tracing (although doctor and nurse responsibility was also recorded for this clinic), and another clinic reported that contact tracing was the responsibility of a combined nurse/health adviser. 


\section{Allergic reaction management}

Only 27 clinics (21\%, range $2-50 \%$ ) reported 48 patients with early syphilis as having penicillin allergy. Only 2 patients, both treated at the same clinic, were de-sensitised to penicillin. Ten clinics reported not having facilities for managing a patient who develops a severe reaction to treatment (anaphylaxis, procaine reaction); nine of these clinics stated having alternative provision for management of severe reaction to treatment (acute hospital services and crash teams)

\section{Discussion}

The preponderance of management of early syphilis in teaching hospitals (about two thirds of cases were managed in teaching hospitals which made up about one quarter of the total number of clinics) reflects their usually larger patient workload and their frequent location in areas of particular need, and highlights the importance of clinics sharing information about practice in regional audit groups.

Although physical examination of the cardiovascular and neurological systems is reported as routinely performed in the investigation of suspected syphilis by over $80 \%$ of clinics, chest radiography is less often performed and lumbar puncture is infrequently carried out. A significant minority of clinics indicated that these examinations and tests are not necessary in early syphilis. There is agreement with the stated use of tests for screening for and investigation of early syphilis between this part of the Audit and the Case Notes Audit with regard to: use of an enzyme immunoassay test by most clinics, more frequent use of the Venereal Diseases Research Laboratory test compared to the rapid plasma reagin test, more frequent use of the treponemal pallidum particle agglutination assay test compared to the treponema pallidum haemagglutination assay test, and little use of polymerase chain reaction and direct fluorescent antibody tests.

Only seven clinics reported using a regimen inferior to that recommended in the National Guidelines $^{3}$ and a further 7 clinics provided no information about treatment of early syphilis. BPG 2.4 gram was the most commonly used treatment, two doses being more commonly used than one. Relatively few clinics reported using PPG treatments in excess of those recommended by the National Guidelines for cases without HIV infection, although this was much more commonly done for cases with HIV infection (29\% of clinics). Nearly all clinics that used doxycycline treatments to treat early syphilis also used PPG and/or BPG regimens. About half of doxycycline treatments 
were with the regimen recommended in the National Guidelines. ${ }^{3}$ The National Guidelines state that treatment of cases with early syphilis and HIV infection is "as for neurosyphilis." However, only 18 clinics specified using the recommended dose and duration (or in excess of this) of PPG for neurosyphilis for cases with HIV infection (and only 10 of these specified using probenecid), and only 2 clinics specified the recommended doxycycline regimen for neurosyphilis.

The results from this part of the Audit serve to illustrate the disparity in results that may be found when both management policies and outcome are audited. The reported use of dark ground microscopy (DGM) by $74 \%$ of clinics does not accord with the actual provision of DGM reported in the Case Notes Audit [32 of $107(30 \%)$ clinics]. ${ }^{2}$ This may be explained by the finding of the British Co-operative Clinical Group survey conducted in 2002 which identified that $35 \%$ of clinics were not confident of their ability to obtain an adequate specimen for DGM, and $64 \%$ were not confident of their expertise to detect treponemes on DGM. ${ }^{4}$ The Bacterial Special Interest Group of BASHH has subsequently developed and delivered a training course on DGM.

Although, all clinics claimed to perform contact tracing, in the Case Notes Audit approximately $15 \%$ of cases were not provided with contact tracing, and $100 \%$ contact tracing was not recorded for any Region. ${ }^{2}$ Likewise, disparity between policy and outcome measures has been reported in the context of another area of sexually transmitted infection management - namely hepatitis B/C infection in patients with HIV infection. ${ }^{5}$

Facilities for managing severe reactions to penicillin treatments appear to be good. However, few patients seem to undergo penicillin desensitisation, despite the fact that this has been shown to be an effective management strategy, including during pregnancy, ${ }^{6}$ and is suggested as a therapeutic option in the National Guidelines. ${ }^{3}$

Acknowledgements are made in the report on Diagnosis and Treatment ${ }^{1}$

Correspondence to: Dr. Hugo McClean, Department of Genitourinary Medicine, Conifer House, West Hull Primary Care NHS Trust, Conifer House, 32-36 Prospect Street, Hull, HU2 8PX. E-mail: Hugo.McClean@whpct.nhs.uk 
1 McClean H, Daniels D, Carne C, Bunting P, Miller R on behalf of the National Audit Group of the British Association for Sexual Health and HIV. UK National Audit of Early Syphilis Management. Case Notes Audit: Diagnosis and Treatment. Int J STD AIDS 200_; _:

2 McClean H, Daniels D, Carne C, Bunting P, Miller R on behalf of the National Audit Group of the British Association for Sexual Health and HIV. UK National Audit of Early Syphilis Management. Case Notes Audit: Contact Tracing, Information giving, Follow-up and Outcomes. Int J STD AIDS 200_; _:

3 British Association for Sexual Health and HIV. Clinical Effectiveness Group. UK National Guidelines on the Management of Early Syphilis 2002 [http://www.bashh.org/guidelines/2002/early\$final0502.pdf] (last accessed 1 May 2005)

4 Rogstad KE, Simms I, Fenton KA, Edwards S, Fisher M, Carne CA. Screening, diagnosis and management of early syphilis in genitourinary medicine clinics in the United Kingdom. Int J STD AIDS 2005;16:348-352

5 Brook MG, Jones K, Dale AWS, Miller RF. Management of HIV and hepatitis B or C coinfection in $15 \mathrm{HIV}$ treatment centres. Disparity between protocols and practice. Int J STD AIDS 2003;14:469-72

6 Ziaya PR, Hankins GD, Gilstrap LC 3rd, Halsey AB. Intravenous penicillin desensitisation and treatment during pregnancy. JAMA 1986;256:2561-2 
Table 1 Test used routinely to screen for and investigate syphilis in UK genitourinary medicine clinics in 2003. $\mathrm{N}=131^{*}$

Test

Treponemal enzyme immunoassay (EIA) IgG, IgM, $\lg \mathrm{G} / \lg \mathrm{M}$

$121(92,65-100)$

Treponema pallidum particle agglutination assay (TPPA)

$96(73,0-100)$

Treponema pallidum haemagglutination assay (TPHA)

$50(38,0-89)$

Fluorescent treponemal antibody absorption (FTA abs)

$56(43,0-100)$

Venereal Diseases Research Laboratory (VDRL)

$100(76,0-100)$

Rapid plasma reagin (RPR)

$46(35,0-100)$

Direct fluorescent antibody (DFA)

$2(2,0-17)$

Polymerase chain reaction (PCR)

$1(1,0-17)$

Dark ground microscopy

$97(74,33-100)$

CSF VDRL

$65(50,11-100)$

CSF RPR

$31(24,0-78)$

CSF white cell count

$73(56,0-86)$

CSF FTA abs

$40(31,0-67)$

CSF TPHA titre

$29(22,0-67)$

CSF TPPA titre

$41(31,0-100)$

${ }^{*}$ More than one choice of routine test was used

$\mathrm{CSF}=$ cerebrospinal fluid 
Table 2 Routine use of parenteral penicillin treatments for early syphilis in different treatment scenarios. Figures are presented as number of clinics using a particular treatment and (national percentages, regional ranges as percentages). $\mathrm{N}=131^{*}$

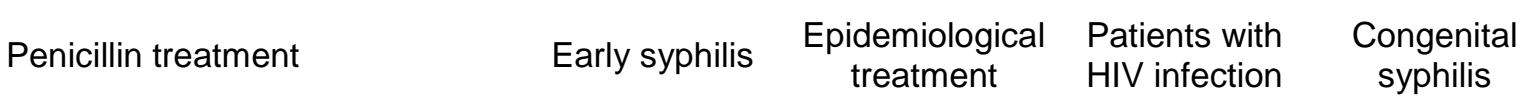

Benzathine penicillin $\mathrm{G} 2.4$ mega units $\times 2$ injections (days 1 \& 8)

Procaine penicillin G $750 \mathrm{mg}$ IM (Jenacillin A $3 \mathrm{ml}$ or Jenacillin $02.5 \mathrm{ml}) \times 10$ days

Benzathine penicillin $\mathrm{G} 2.4$ mega units $\times 1$ injection IM

$$
75(57,0-100)
$$$$
28(21,0-56)
$$

$28(21,0-100)$

$5(4,0-22)$

$$
52(40,0-75)
$$$$
13(10,0-50)
$$

$20(15,0-56)$

$51(39,0-100)$

$5(4,0-33)$

$1(1,0-11)$

Benzathine penicillin $\mathrm{G} 2.4$ mega units $x 3$ injections IM

$$
2(2,0-11)
$$$$
1(1,0-8) \quad 18(14,0-100)
$$

Long-acting biclinocillin 2 mega units IM (containing benethamine1.2 mega units)

Long-acting procaine penicillin $\mathrm{G}$ in aluminium stearate (Jenacillin O) 2 mega units IM

Benzyl penicillin G sodium 100,000-

150,000 units/kilogram IV 12 hourly for first 7 days of life and 8 hourly thereafter for 10 days ${ }^{\ddagger}$

0 (NA)

$2(2,0-11)$

0 (NA)

$2(2,0-17)$

$$
0 \text { (NA) }
$$

$$
0 \text { (NA) }
$$

$$
0 \text { (NA) }
$$

Procaine penicillin G 50,000 units/kilogram IM for 10 days (Jenacillin $A 0.2 \mathrm{ml} / \mathrm{kg}$ )

\section{END.}

